\title{
Effects of a patient oriented decision aid for prioritising treatment goals in diabetes: pragmatic randomised controlled trial
}

\author{
(c) $\frac{(1)(8)}{\text { gy }}$ OPC OPEN ACCESS
}

\author{
Petra Denig professor of drug utilisation quality ${ }^{12}$, Jan Schuling general practitioner trainer ${ }^{2}$, Flora \\ Haaijer-Ruskamp professor in drug utilisation studies ${ }^{12}$, Jaco Voorham assistant professor clinical \\ pharmacology ${ }^{12}$
}

${ }^{1}$ Clinical Pharmacy and Pharmacology, University Medical Center Groningen, PO Box 30.001, Groningen, Netherlands; ${ }^{2}$ University of Groningen, University Medical Center Groningen, Groningen, Netherlands

\begin{abstract}
Objective To assess the effects of a patient oriented decision aid for prioritising treatment goals in diabetes compared with usual care on patient empowerment and treatment decisions.

Design Pragmatic randomised controlled trial.

Setting 18 general practices in the north of the Netherlands.

Participants 344 patients with type 2 diabetes aged $\leq 65$ years at the time of diagnosis and managed in primary care between April 2011 and August 2012: 225 were allocated to the intervention group and 119 to the usual care group.

Intervention The intervention comprised a decision aid for people with diabetes, with individually tailored risk information and treatment options for multiple risk factors. The aid was intended to empower patients to prioritise between clinical domains and to support treatment decisions. It was offered to participants before a regular diabetes check-up and to their healthcare provider during the consultation. Four different formats of the decision aid were included for additional explorative analyses.
\end{abstract}

Main outcome measures The primary outcome was the effects on patient empowerment for setting and achieving goals. The secondary outcomes were changes in the prescribing of drugs to regulate glucose, blood pressure, lipids, and albuminuria. Data were collected through structured questionnaires and automated data extraction from electronic health records during six months before and after the intervention.
Results Of all intervention participants, 103 (46\%) reported to have received the basic elements of the intervention. For the primary outcome analysis, 199 intervention and 107 control patients with sufficient baseline and follow-up data could be included. The mean empowerment score increased 0.1 on a 5 point scale in the overall intervention group, which was not significantly different from that of the control group (mean difference after adjusting for baseline $0.039,95 \%$ confidence interval -0.056 to 0.134$)$. Lipid regulating drug treatment was intensified in $25 \%$ of intervention and $12 \%$ of control participants with increased cholesterol levels, which did not reach significance when the intervention was compared with the usual care group (odds ratio $2.54,95 \%$ confidence interval 0.89 to 7.23 ). Prespecified explorative analyses showed that this effect was significant for the printed version of the decision aid in comparison to usual care $(3.90,1.29$ to 11.80$)$. No relevant or significant changes were seen for other treatments.

Conclusion We found no evidence that the patient oriented treatment decision aid improves patient empowerment by an important amount. The aid was not used to its full extent in a substantial number of participants.

Trial registration Dutch trial register NTR1942.

\section{Introduction}

Adequate treatment targeting multiple risk factors can prevent or slow the progression of complications in people with diabetes. ${ }^{12}$ Despite improvements observed in several processes

Correspondence to: P Denig p.denig@umcg.nl

Extra material supplied by the author (see http://www.bmj.com/content/349/bmj.g5651?tab=related\#datasupp)

Example of graph showing potential risk reduction for patients

Examples of treatment cards

Supplementary table 1

Supplementary table 2

Supplementary table 3

Supplementary table 4

Supplementary table 5 
of diabetes care in the past decade, the control of risk factors remains suboptimal..$^{3-5}$ Two modifiable factors have been identified, which are likely to contribute to these suboptimal results. ${ }^{67}$ Firstly, clinicians are sometimes hesitant to intensify treatment in people with increased levels of risk factors. ${ }^{8}$ In addition, patients may show poor adherence to drugs, ${ }^{9}$ which is not always acknowledged by their clinician..$^{11}$

Models of chronic care emphasise the need for a collaborative approach between patient and healthcare providers to achieve effective disease management. ${ }^{12}{ }^{13}$ They must work together to identify problems, set priorities, establish goals, and make treatment plans. ${ }^{14}{ }^{15}$ A proactive role by patients may also result in a more timely adjustment of drugs by their doctors. ${ }^{16} 17$ Although the concept of a collaborative approach is widely acknowledged, it is not yet an integral part of daily practice. ${ }^{18}$

Decision aids or support systems can encourage patient-provider discussions about disease management by presenting available treatment options and expected outcomes for each patient. ${ }^{19}$ Most aids, however, focus on one specific treatment choice, whereas people with diabetes are often confronted with multiple clinical domains (glucose, blood pressure, lipids) for which treatment is indicated. The newest generation decision aids prioritise between domains to support treatment decisions, but their effect when used jointly by patient and healthcare provider has not yet been evaluated. ${ }^{20} 21$

We present the results of a pragmatic randomised controlled trial of a newly developed patient oriented decision aid for prioritising treatment goals in diabetes. Our primary aim was to assess the effect of the decision aid compared with usual care on patient empowerment for setting and achieving goals. We expected that more empowerment would translate as patients getting more involved in the process of goal setting and decision making to achieve those goals and subsequently in intensified drug treatment in patients not achieving goals. We anticipated that the intervention could also lead to potentially negative effects on other patient outcomes. Our secondary aim was to explore the impact of different presentation formats and modifying effects of patient characteristics and to learn more about how the aid was used in daily practice.

\section{Methods}

\section{Study setting and recruitment of practices}

A total of 18 general practices were recruited in the north of the Netherlands. In this region, a diabetes disease management programme was implemented in 2007. As part of this programme, each practice receives a performance report yearly. All general practices use electronic medical record systems supporting structured care protocols and were eligible for inclusion. Most practices have a nurse practitioner or specialised assistant for diabetes care who carries out the quarterly diabetes checks and is trained to conduct physical examinations, risk assessments, patient education, and counselling. Changes in drug treatment can be proposed by such staff but have to be approved by the general practitioner.

To ensure a comparable level of communication skills we offered all the practices a training course in motivational interviewing before the study started. If they had already been on such a course, the practices were offered $€ 250$ (£199; \$324) as incentive to participate. In addition, all participants received a two hour training session in risk communication, including role play with simulation patients and an instruction video with simulated consultations showing "good" and "bad" examples of applying four basic principles of risk communication-that is, to use natural frequencies, positive and negative phrasing, and explicit uncertainty, and to be open and refrain from imposing options.

\section{Patient population and recruitment}

We included people with type 2 diabetes who were managed in primary care. We excluded patients who had had a myocardial infarction in the preceding year; experienced a stroke; had heart failure, angina pectoris, or a terminal illness; and were aged more than 65 years at the time their diabetes was diagnosed. For these patients the calculated risks using the intervention tool were not considered sufficiently evidence based. There was no restriction on duration of diabetes or age at time of inclusion. After we had identified potentially eligible patients from the electronic medical record system, we presented a random selection of at least 40 patients per practice to the healthcare provider, who was asked to confirm the eligibility criteria and exclude patients with dementia, cognitive deficits, blindness, or an inability to read Dutch. We chose this procedure since these exclusion criteria could not be reliably identified from the medical records. We used random selections because in large practices we did not want to burden healthcare providers with the verification of all potentially eligible patients. Between April 2011 and August 2012, these patients received an information package distributed by their healthcare provider and containing an invitation letter, information about the project, and an informed consent form. Patients were offered $€ 10$ for time spent on completing the study questionnaires.

\section{Intervention}

We developed a decision aid for people with diabetes, which presents individually tailored information on risks and treatment options for multiple risk factors. Specific risk factors included HbA1c, systolic blood pressure, low density lipoprotein cholesterol, and smoking. The rationale, goals, and a detailed description of the decision aid have been presented previously. ${ }^{22}$ In short, the aid focuses on shared goal setting and decision making, particularly with respect to the drug treatment of risk factors. We followed a stepwise development process, including active involvement and testing with patients and providers. ${ }^{22}$ The decision aid shows several graphs using individually tailored information. Risks calculated with the United Kingdom Prospective Diabetes Study risk score were explained in positive and negative terms and expressing uncertainty. For example, "Out of 100 patients with the same age, gender, and disease characteristics as you, 16 are expected to get a heart attack within the next five years and 84 will not get a heart attack. At this moment, we cannot say to which group you will belong." Graphs are then presented showing potential risk reductions with possible treatment options and questions posed to the patient (see supplementary figure $1 \mathrm{and}^{22}$ ).

Key features, identified as being relevant for productive patient-provider interaction, included a personal status report including test results and current drug treatment; the presentation of tailored information on achievable treatment goals and possible treatment options for specific risk factors; a combination of graphs and text using natural frequencies for outcome probabilities; the presentation of pros and cons of all treatment options; and asking patients to think about treatment options. ${ }^{22}$ The aid retrieves clinical information directly from the electronic medical record. It should be used by patients before a regular quarterly check-up and discussed jointly with their healthcare provider during the consultation to help them prioritise on treatment that will maximise relevant outcomes. The software is integrated in the electronic medical record 
system, making use of all available information but also allowing for additional data entry or corrections. The software is complemented with a set of treatment cards that can be used during consultation, summarising the positive and side effects of the various treatment options, including doing nothing (see supplementary fig 2).

A short instruction protocol was provided on how to use the software and treatment cards before and during a consultation. ${ }^{22}$ To summarise, before the consultation the healthcare provider needed to start the software, check the content, and print the information. The patients were asked to come to the practice 15 minutes in advance to go through the information, either in print or on the computer. During the consultation, healthcare providers were expected to support patients to think about treatment goals and options, making use of the computer screen or printed version of the information. When appropriate, healthcare providers could present and compare specific treatment options using the treatment cards. The consultation ideally was to be concluded with clear action points. At the end of the consultation, the printed version was to be distributed to all intervention patients. When the regular scheduled time was too short, a further consultation could be planned to finalise the shared decision making.

Patients in the control group received care as usual-that is, their regular quarterly check-up, including any education, information, or additional consultations as deemed necessary by their healthcare provider.

\section{Design and treatment allocation}

A primary analysis was conducted to assess the effect of the decision aid compared with usual care. To explore the impact of different formats, we included four versions for presenting the information to patients in a $2 \times 2$ factorial design: a computer screen or printed version, presented either as a short version, showing treatment effects on myocardial infarction risk only, or as an extended version, including effects on additional outcomes (stroke, amputation, blindness, renal failure).

We randomly allocated participating practices to the computer screen or printed version. A stratified computer generated allocation sequence was used, defining four strata by practice size ( $<2500$ patients or $>2500$ patients) and organisation type (single general practitioner or several general practitioners). Within each practice, consenting patients were randomised to receive the short version presenting only myocardial infarction risk outcomes, the extended version presenting additional outcomes, or to the control group. We used a predefined computer algorithm with a blockwise scheme to conceal the allocation process from the healthcare provider.

\section{Outcome measures}

Our primary outcome was the empowerment of patients for setting and achieving goals, as assessed with the diabetes empowerment scale - subscale for setting and achieving diabetes goals (DES-III). ${ }^{23}$ This instrument can be downloaded from the website of the Michigan Diabetes Research and Training Center (www.med.umich.edu/mdrtc/profs/survey.html\#des).

Furthermore, we assessed changes in treatment. This included measures assessing intensification of drug treatment for patients with increased $\mathrm{HbA} 1 \mathrm{c}$ levels, systolic blood pressure, or low density lipoprotein cholesterol levels, and treatment with renin angiotensin system inhibiting agents. In addition, we explored the effects of the decision aid on secondary patient outcomes. These were:
- other diabetes self efficacy aspects, as measured with the diabetes empowerment subscales for managing the psychosocial aspects of diabetes (DES-I), and assessing dissatisfaction and readiness to change (DES-II)

- beliefs about medicines, as measured with the beliefs about medication questionnaire-subscales necessity, concerns, general overuse, and general harm ${ }^{24}$

- satisfaction with diabetes care, as measured with the patients' evaluation of quality of diabetes care questionnaire $^{25}$

- negative emotions, as measured with the problem areas in diabetes questionnaire ${ }^{26}$

- general health status, as measured with the Dutch tariff of the EQ-5D ${ }^{27}$

- smoking status.

\section{Effect modification}

Effects of the decision aid may depend on characteristics of the patients that affect their involvement, understanding, and patient-provider communication about disease management. Therefore, we tested several characteristics as possible modifying factors: age, low educational level (representing not more than primary school or lower vocational education), short duration of diabetes ( $<3$ years), number of uncontrolled risk factors (0-4 scale for HbA1c, systolic blood pressure, low density lipoprotein cholesterol, smoking), a high number of related drugs at baseline ( $>3$ glucose, blood pressure, or lipid regulating drugs), or polypharmacy ( $>4$ drugs for chronic illnesses) at baseline.

\section{Data collection}

We used structured questionnaires to collected patient survey data, which were mailed to the patients in the month before and three to four months after the scheduled quarterly check-up. In the post-intervention questionnaire, intervention patients rated how easy or difficult they perceived the decision aid on a 5 point Likert scale, which we re-coded into a binary difficulty score (yes=difficult, no=neutral or easy). We collected data on patient characteristics, smoking status, and drugs in the six months before and after the intervention period from the GIANTT (Groningen initiative to analyse type 2 diabetes treatment) database. This database includes longitudinal data collected from primary care medical records using validated automated extraction procedures. ${ }^{28}$ Diabetes duration was based on information provided by the healthcare provider. We calculated the United Kingdom Prospective Diabetes Study risk score and the number of uncontrolled risk factors at baseline using the last values for $\mathrm{HbA} 1 \mathrm{c}$, blood pressure, cholesterol, and smoking status in the year before the consultation. Since no valid information on ethnicity was available, and more than $90 \%$ of the population in the study region is white, we used "white" ethnicity for calculation of the risk score. ${ }^{29}$

\section{Process evaluation}

In the post-intervention questionnaire, intervention patients were asked whether they received the aid before their consultation, discussed the cardiovascular risk during the consultation with their healthcare provider, and discussed how such risks could be reduced. For the extended version, patients answered one additional question: whether they also discussed other diabetes related risks. This information was used for a per protocol analysis where we included only patients who answered 
yes to all questions. In addition, healthcare providers were asked to complete a short checklist after each consultation with an intervention patient about their perception of how the decision aid was used before and during the consultation.

\section{Sample size}

The sample size was preliminary estimated at 150 patients per study arm to achieve a power of $80 \%$ at a $5 \%$ significance level for detecting a 0.2 absolute difference in the diabetes empowerment score, which was the primary outcome, between the overall intervention and control group. ${ }^{22}$ Since there is no published minimum level of relevant change in the diabetes empowerment subscale ${ }^{30}$ and the standard deviation in the empowerment score at baseline was smaller than assumed for this preliminary estimation, we conducted a retrospective power calculation after all patients were included and baseline data were collected. With a total number of 199 patients in the intervention group and 107 in the control group for the primary outcome analysis and an observed standard deviation of 0.46 for the empowerment score, we achieved an $80 \%$ power at $5 \%$ significance level to detect a difference in the empowerment score of 0.15

\section{Statistical analysis}

We used descriptive statistics to summarise personal, clinical, and other patient outcomes and calculated standardised differences to check for imbalance between the intervention and usual care group. ${ }^{31}$ The effects of the intervention were tested in an intention to treat analysis comparing the patient groups not adjusted for clustering. We assumed that the effect of the decision aid on patient empowerment was mainly influenced by patient and not by practice characteristics. To check this, we conducted a multilevel analysis to estimate whether the practice level had an impact on the primary outcome. The proportion of variance that was accounted for at this level was calculated as the intraclass correlation coefficient. This coefficient was 0.059 for the primary patient outcome, indicating that there was not much variance due to practice related factors (see supplementary table 1). We tested effects using linear and logistic regression models, adjusting for baseline values of the outcome of interest. We present regression coefficients as the effect size measure for the linear models, indicating the mean difference between the intervention and control group after adjusting for baseline. For the logistic regression models, we present odds ratios with $95 \%$ confidence intervals. Imbalance, which was defined as a standardised difference of more than 0.1 , was observed in some of the variables, indicating that randomisation was not fully successful. Because of this imbalance, we conducted additional analyses adjusting for unbalanced baseline patient characteristics. We used multiple imputation by chained equations to impute missing baseline values for risk factors and educational level. Results were combined across 20 imputed datasets using Rubin's rules. ${ }^{32}$ We evaluated interactions of the intervention with each of the effect modifiers. Finally, explorative analyses were conducted to compare the effects for each of the intervention formats with the usual care group separately, and to compare the computer screen with the printed version, as well as the short with the extended version. Data were analysed using Stata version 12.

\section{Results}

The 18 included general practices had an average patient population of 4380 . Eleven practices had one nurse practitioner, nurse, or specialised assistant for diabetes care, six had two or three nurse practitioners or specialised assistants, and one had no such staff. Together these were the 25 healthcare providers who conducted the quarterly diabetes check-ups and therefore worked with the decision aid and received the training on risk communication using the aid. Our stratified randomisation scheme based on practice size and number of general practitioners resulted in an equal distribution of the printed and computer screen version among practices with one or more than one healthcare provider working with the decision aid.

Of the people with diabetes and identified as potentially eligible in the electronic medical records of the 18 practices, 1769 were assessed for exclusion criteria (figure $\Downarrow$ ). Of the resulting 665 eligible patients, 370 (56\%) consented to participate. For 344, a practice consultation was anticipated during the study intervention period, and these patients were randomised to the intervention $(n=225)$ or usual care group $(n=119)$. The intervention patients were comparable to usual care patients for age, sex, and educational level (table $1 \Downarrow$ ). Although being treated less often with insulin and more often with sulphonylurea derivatives and other oral glucose regulating drugs, more intervention than usual care patients had a well controlled $\mathrm{HbA1c}$ level at baseline. On the other hand, more usual care patients had a well controlled blood pressure level at baseline. Overall, however, the groups were comparable for the number of uncontrolled risk factors and the number of drugs used at baseline. Only $15 \%$ of the intervention patients and $18 \%$ of the usual care patients showed adequate control for all risk factors. Intervention patients had slightly lower empowerment scores, higher beliefs about the necessity of their treatment, and a lower general health status (table 1).

\section{Use of the decision aid}

The decision aid was accessed at the consultation date for 198 of the 225 intervention patients; for eight no session was planned within the study period, for seven the aid was accessed after the planned consultation date, and for 12 the session failed to be logged because of technical problems. In total, 103 patients reported to have received all basic elements of the intervention. These patients were significantly younger ( $60.0 v 63.6$ years), had higher HbA1c levels (6.9\% v 6.7\%), and more uncontrolled risk factors (on average $1.5 v 1.3$ ) at baseline than patients who reported not receiving the full intervention. They were similar for sex, educational level, drug treatment, and baseline responses to the questionnaires (see supplementary table 2).

\section{Effect on patient reported outcomes}

Baseline patient empowerment scores were available for 212 intervention and 110 usual care patients (table 1), whereas outcomes scores were available for 207 and 108 patients, respectively (table $2 \Downarrow$ ). The average empowerment score for setting and achieving goals increased by 0.1 on a 5 point Likert scale among the patients in the intervention group, which was not significantly different from the usual care group (effect size $0.039,95 \%$ confidence interval -0.056 to 0.134 , table 2 ). No significant effects were observed for any of the other patient reported outcomes, including satisfaction with diabetes care, negative emotions, and general health status.

Adjustment on unbalanced patient characteristics did not change the effect of the intervention on empowerment for setting and achieving goals (effect size $0.045,95 \%$ confidence interval -0.055 to 0.139 , see supplementary table $3 a$ ). The results for the per protocol analysis were similar $(0.058,-0.055$ to 0.172 , see supplementary table 3b). Age, educational level, duration of diabetes, number of uncontrolled risk factors, and number of 
drugs used at baseline did not modify the effect of the intervention on empowerment (see supplementary table 4).

\section{Effect on treatment}

In patients not reaching the low density lipoprotein cholesterol target of $2.5 \mathrm{mmol} / \mathrm{L}$, lipid regulating treatment was intensified in $25 \%$ of the intervention patients compared with $12 \%$ of the usual care patients. This effect was not significant (odds ratio $2.54,95 \%$ confidence interval 0.89 to 7.23 , table $3 \Downarrow$ ). No differences were seen for intensification of drug treatment in patients not reaching $\mathrm{HbAlc}$ or blood pressure targets or in the prescribing of renin angiotensin system inhibiting agents.

Results were similar on adjustment of unbalanced patient characteristics and for the per protocol analysis (see supplementary tables $3 \mathrm{a}$ and $\mathrm{b}$ ). The effect of the intervention was not significantly modified by age, level of education, duration of diabetes, number of uncontrolled risk factors, and number of drugs used at baseline (see supplementary table 4).

\section{Different presentation formats}

None of the presentation formats of the decision aid showed a significant effect on patient empowerment (see supplementary table 5). More intensified lipid regulating treatment was seen for patients who received the printed version compared with usual care (odds ratio 3.90, 95\% confidence interval 1.29 to 11.80), and comparing the computer screen with the printed version $(0.34,0.12$ to 0.98 , see supplementary table 5$)$. Differences in intensified blood pressure and glucose regulating treatment, favouring the printed and the extended version, respectively, were only observed when these formats were compared with each other but not when compared with usual care (see supplementary table 5).

\section{Evaluation by patients}

For all four presentation formats, more than three quarters of the patients reported receiving the decision aid before the consultation (table $4 \Downarrow$ ). Discussion of risks and possible risk reductions was lowest in the group receiving the extended screen version. These patients also had the lowest number of uncontrolled risk factors and risk score (table 4). Only 10 patients perceived the information as difficult, including seven who had received the extended version of the aid. Most patients who received the computer screen version before the consultation also received the printed version during or after the consultation as planned. Almost 30\% reported that they preferred the printed version over the screen version, whereas most of the others did not have a preference (table 4).

\section{Quantitative evaluation by healthcare providers}

For 185 of the 217 intervention consultations (85\%), the healthcare providers completed the checklists after the consultations about the decision aid and the decision making process. Two general practices, both randomised to the printed version, did not complete these checklists. According to the healthcare providers, almost all patients received the aid before the consultation. This included 26 patients $(15$ computer screen group, 11 printed version group) who reported not having seen the aid. Patients in the computer screen group were more often assisted by practice staff than patients in the printed version group (table 4). The providers reported that they discussed possible risk reductions for almost all patients. The 15 reported cases where they did not do this, concerned patients with a lower overall cardiovascular risk (mean risk score $4.2 v 7.2$ ). In more than two thirds of the consultations the providers reported that they had involved the patient in the treatment decisions (table 4). Additional consultations were planned in 16 of the 185 cases (9\%). For 75 consultations, the healthcare provider made a specific remark about the use of the decision aid (table $5 \Downarrow$ ). Most were positive remarks related to patient experiences $(n=32)$, who perceived the information as useful, clear, reassuring, or motivating. There were 10 reports of patients who had been encouraged by the aid to improve their lifestyle, and four who had been motivated to disclose that they had stopped taking a drug or were willing to start again. The providers reported that 13 patients had been concerned or reluctant, and seven had been irritated by the information. The healthcare providers perceived the information as not needed for eight patients and not motivating for lifestyle problems for another eight patients. For five patients, there were some practical concerns. Finally, six patients reported difficulties in completing the pre-intervention questionnaires.

\section{Discussion}

Our study does not provide evidence that a patient oriented treatment decision aid dealing with multiple clinical domains for people with diabetes improves patient empowerment for setting and achieving goals by a important amount. Only $46 \%$ of the intervention patients reported that they had received the basic elements of the intervention. No consistent changes were observed in other patient reported outcomes, such as satisfaction with care, beliefs about drugs, negative emotions, or general health status. In general, no significant changes were seen for drug treatment. The printed version of the intervention may have increased lipid regulating treatment in poorly controlled patients. Most patients did not perceive the information as difficult. For some patients, the healthcare providers perceived the information as not needed or not motivating.

\section{Strengths and weaknesses of this study}

We developed a decision aid using a theoretical framework, following the International Patient Decision Aids Standards, and tested its effectiveness in a randomised trial conducted in a routine practice setting. ${ }^{33}{ }^{34}$ The aid was developed to fit in with the workflow in Dutch primary care and deal with many of the drawbacks of previously developed decision aids. ${ }^{22}$ It can be characterised as a minimal intensity strategy that requires little extra time to conduct. We provided a short training session in risk communication with the aid for healthcare providers but no formal assessment was done on whether they were able to engage patients in a dialogue. To be included, however, they needed to have followed a course in motivational interviewing to ensure a comparable level of training in communication skills in all practices.

The most obvious weakness was the limited use of the decision aid. In our study, the aid was used before and during only one regular diabetes check-up. One might question whether single exposure to an intervention can substantially change patient empowerment. For chronic diseases, a decision aid should ideally be used when needed and repeatedly over time. Exposure seems to be a relevant factor when involving patients in tailored interventions, ${ }^{35}$ and more effect of the aid could be anticipated when it is used more often in the course of disease management. Also, the healthcare providers were not accustomed to using the decision aid, and their level of motivation and expertise may improve with routine use of the aid in daily practice. We did not assess what actually happened during the consultations but evaluated the use of the aid in post-intervention questionnaires. 
The healthcare providers sometimes perceived the information as unneeded. It seemed that the decision aid was not used as intended in a substantial number of the patients, who were on average somewhat older and had fewer uncontrolled risk factors.

We expected that increased patient empowerment would translate as more patient involvement in setting and achieving treatment goals. This could lead to more but also less intensified treatment, depending on the patient's goals. Given the existing levels of suboptimal treatment, however, we expected that in general the decision aid would lead to intensified drug treatment. We included four measures assessing changes in drug treatment. Observing a change in only one of them for the printed version of the aid could be a chance finding. In the post-intervention interviews, however, it was explicitly mentioned that some patients had been motivated by the intervention to start using a drug.

Patients participating in this study were on average about five years younger but had a similar duration of diabetes to the population of people with type 2 diabetes managed in Dutch primary care. ${ }^{45}$ Nevertheless, the age distribution was wide and the effect of the intervention seemed not to be affected by age. Although most patients included in our study showed some potential for improved management of their risk factors, the risk factors were on average relatively well controlled. Participating practices may be more open to shared decision making and already have a high level of chronic disease management.

We chose a pragmatic design, evaluating the effect of the intervention between patient groups since this was a first test of a novel type of decision aid. If the aid is found to be beneficial, it can be tested on a larger scale, also taking practice level factors into account. Our design with patients randomised within practices increases the risk of contamination in the control group, and thus decreases the power to detect the effects of the intervention. Both patients and providers may be affected, resulting in improvements in the usual care group from study participation. This design, however, was deemed necessary to ensure that variations in communication skills and practice organisation were balanced between the intervention and usual care groups. In addition, when practices are randomised to a non-intervention arm it may induce low participation and high drop-out rates. To prevent direct contamination, the decision aid could not be used for patients other than the intervention patients. None the less, it was possible that healthcare providers used some of the aspects learnt from working with the novel approach in their usual care. We observed some differences in clinical and patient reported outcomes between intervention and control patients, indicating that our randomisation did not fully work. Therefore, we conducted additional analysis incorporating adjustment on unbalanced baseline characteristics, but this did not change our main conclusions.

\section{Strengths and weaknesses in relation to other studies}

So far, only a few computer based or web based disease management systems have been tested that offer tailored status reports with reminders or recommendations for individual patients. ${ }^{36}$ This is an area that is rapidly evolving owing to the increased attention for patient empowerment as well as improving patient-provider communication and shared decision making. Implementing such supportive systems has become more feasible in various settings owing to the increasing use of electronic medical records and internet access. Effective communication involves patients receiving tailored information and being encouraged to express concerns about the recommended treatment. ${ }^{38}$ Recently, one study described the development of a web based tool designed to facilitate the articulation of patients' often unvoiced agendas, which patients have to complete before a consultation with their diabetologist. ${ }^{39}$ Our study is one of the first to evaluate the impact of such an intervention.

In people with poorly controlled diabetes, computer or web based aids have shown potential to improve HbA1c levels. ${ }^{36} 37$ Although a decision aid could also motivate well controlled patients to maintain optimal control, these patients are often excluded from these studies. Furthermore, most decision aids that have been developed and tested for people with diabetes focus on healthy behaviour and self monitoring skills and not on drug treatment. ${ }^{40}{ }^{41}$ Recently, some aids have been developed that also present drug treatment as a possible option to patients but their effects have not yet been evaluated. ${ }^{20}{ }^{21}$ One exception is the paper based "diabetes medication choice" aid, which can be used during consultation to encourage patients to voice their opinions about drug options to the clinician. ${ }^{42}$ This aid was found to be effective for involving those patients who were eligible for a treatment change in the decision-making process. ${ }^{33} 43$ Although the healthcare providers stated that they provided and discussed the information in the majority of the consultations, more than half of the patients reported not having received it as planned. It is possible that in some of these cases the providers only discussed the information briefly. Previously, it was found that healthcare providers underestimate the level of involvement that patients want, and providers have difficulties estimating the information needs of people with diabetes. ${ }^{44} 45$ This may contribute to an overly optimistic view of a shared decision -making process.

Few studies have looked at possible negative effects of decision aids for people with diabetes. We included several secondary patient outcomes to provide insight into how aid may affect diabetes care and patient outcomes beyond its primary aim. We observed no apparent negative effects of our decision aid. There were no changes in satisfaction with care or general health outcomes. This finding is consistent with the conclusion of a recent Cochrane review about the effects of decision aids for people facing health treatment or screening decisions. ${ }^{19}$ In addition, we did not see any adverse effects in diabetes related negative emotions.

\section{Meaning of the study}

Our study showed that a patient oriented treatment decision aid dealing with multiple clinical domains for people with diabetes did not significantly increase patient empowerment for setting and achieving goals in Dutch primary care. The printed version of our intervention showed some effect on lipid regulating treatment for patients with poorly controlled low density lipoprotein cholesterol. Given our multiple testing, this could be a chance finding. We can only speculate why no changes were seen for blood pressure and glucose regulating treatment. Possibly, the fact that most patients were already prescribed one or more drugs for these risk factors limited the options or willingness for additional drug treatment. In our setting, mostly nurse practitioners and specialised assistants conducted the consultations using the aid. We do not know whether this affected its impact.

Our finding that the decision aid was not used to its full extent in a substantial number of patients is a matter of concern. These patients could not remember receiving or discussing the decision aid. They were more often patients who were well controlled 
or had a low risk for cardiovascular complications. Given the comments of several healthcare providers that they sometimes perceived the information as unneeded, the aid may be more effective when it is used more selectively. A flexible method, where the aid can be offered to all patients before the consultation but used selectively during consultation, might be a sensible approach for routine care. The printed version seemed to show more effect than the computer screen version. Several patients expressed a preference for the printed version.

Healthcare providers mentioned that patients more often needed assistance when using the computer screen version. Considering the average age and educational level of the current population with diabetes, printing the tailored information seems to be the preferable choice at present.

\section{Future research}

Several problems have not yet been tackled. Firstly, the intervention may have motivated patients to improve their drug taking behaviour, which was not assessed in our study. A follow-up study will be conducted to assess the impact of the intervention on several clinical outcomes. When patients improve their drug taking behaviour, this can be reflected in better clinical outcomes. Secondly, qualitative interviews with the healthcare providers were conducted after completion of the intervention study to collect their experiences with the decision aid in more depth. A future study examining these additional data may help to identify relevant elements and barriers for effective use of the aid. Finally, future studies are needed to evaluate effects of repeated and more tailored use of the aid applied in routine care, and studies could be conducted using similar decision aids in other patient populations and settings.

Contributors: PD, FH-R, and JV designed the study and formulated the research questions. PD, JS, and JV developed and tested the decision aid. All authors researched and interpreted the data. PD drafted the manuscript. FH-R, JS, and JV critically revised and edited the manuscript: All authors read and approved the final manuscript. The funders had no role in the study design, the data collection, analysis and interpretation, the writing of the report, nor the decision to submit the article for publication. All authors had full access to all of the data in the study and can take responsibility for the integrity of the data and the accuracy of the data analysis. PD is the guarantor of this study.

Funding: This study has been funded by ZonMW—-the Netherlands Organisation for Health Research and Development.

Competing interests: All authors have completed the ICMJE uniform disclosure form at www.icmje.org/coi_disclosure.pdf and declare: no support from any organisation for the submitted work; no financial relationships with any organisations that might have an interest in the submitted work in the previous three years; no other relationships or activities that could appear to have influenced the submitted work.

Ethical approval: This study was approved by the medical ethics committee of the University Medical Center Groningen (ABR No NL 29042.042.09).

Data sharing: Patient level data are stored within the GIANTT database at the University Medical Centre Groningen. Research team members have been granted access to the required data by the GIANTT steering committee. Presented data are anonymised and risk of identification is low. No consent for data sharing with other parties was obtained but the corresponding author may be contacted to forward requests for data sharing.

Transparency: The lead author (PD) affirms that the manuscript is an honest, accurate, and transparent account of the study being reported. No important aspects of the study have been omitted.
1 Gaede P, Vedel P, Larsen N, Jensen GVH, Parving H, Pedersen O. Multifactorial intervention and cardiovascular disease in patients with type 2 diabetes. $N$ Engl $J$ Med 2003;348:383-93.

2 Buse JB, Ginsberg HN, Bakris GL, Clark NG, Costa F, Eckel R, et al. Primary prevention of cardiovascular diseases in people with diabetes mellitus. Diabetes Care 2007;30:162-72.

3 Voorham J, Haaijer-Ruskamp FM, van der Meer K, de Zeeuw D, Wolffenbuttel BHR, Hoogenberg K, et al. Identifying targets to improve treatment in type 2 diabetes; the Groningen Initiative to aNalyse Type 2 diabetes Treatment (GIANTT) observational study. Pharmacoepidemiol Drug Saf 2010;19:1078-86.

4 Sidorenkov G, Haaijer-Ruskamp FM, de Zeeuw D, Denig P. A Longitudinal study examining adherence to guidelines in diabetes care according to different definitions of adequacy and timeliness. PLoS One 2011;6:e24278.

5 Van Hateren KJ, Drion I, Kleefstra N, Groenier KH, Houweling ST, van der Meer K, et al. A prospective observational study of quality of diabetes care in a shared care setting: trends and age differences (ZODIAC-19). BMJ Open 2012;2(4).

6 Schmittdiel JA, Uratsu CS, Karter AJ, Heisler M, Subramanian U, Mangione CM, et al. Why don't diabetes patients achieve recommended risk factor targets? Poor adherence versus lack of treatment intensification. J Gen Intern Med 2008;23:588-94.

7 Handelsman $\mathrm{Y}$, Jellinger PS. Overcoming obstacles in risk factor management in type 2 diabetes mellitus. J Clin Hypertens 2011;13:613-20.

8 Voorham J, Haaijer-Ruskamp FM, Stolk RP, Wolffenbuttel BH, Denig P; Groningen Initiative to Analyze Type 2 Diabetes Treatment Group. Influence of elevated cardiometabolic risk factor levels on treatment changes in type 2 diabetes. Diabetes Care 2008;31:501-3.

9 Vink NM, Klungel OH, Stolk RP, Denig P. Comparison of various measures for assessing medication refill adherence using prescription data. Pharmacoepidemiol Drug Saf 2009:18:159-65.

10 Meddings J, Kerr EA, Heisler M, Hofer TP. Physician assessments of medication adherence and decisions to intensify medications for patients with uncontrolled blood pressure: still no better than a coin toss. BMC Health Serv Res 2012;12:270.

11 Voorham J, Haaijer-Ruskamp FM, Wolffenbuttel BH, Stolk RP, Denig P; Groningen Initiative to Analyze Type 2 Diabetes Treatment Group. Medication adherence affects treatment modifications in patients with type 2 diabetes. Clin Ther 2011;33:121-34.

12 Von Korff M, Gruman J, Schaefer J, Curry SJ, Wagner EH. Collaborative management of chronic illness. Ann Intern Med 1997;127:1097-102.

13 Wagner EH, Austin BT, Davis C, Hindmarsh M, Schaefer J, Bonomi A. Improving chronic illness care: translating evidence into action. Health Aff 2001;20:64-78.

14 Montori V, Gafni A, Charles C. A shared treatment decision-making approach between patients with chronic conditions and their clinicians: the case of diabetes. Health Expect 2006;9:25-36.

15 Joosten EAG, DeFuentes-Merillas L, de Weert GH, Sensky T, van der Staak CPF, de Jong CAJ. Systematic review of the effects of shared decision-making on patien satisfaction, treatment adherence and health status. Psychother Psychosom 2008;77:219-26.

16 Rachmani R, Slavacheski I, Berla M, Frommer-Shapira R, Ravid M. Treatment of high-risk patients with diabetes: motivation and teaching intervention. J Am Soc Nephrol 2005;16:S22-6.

17 Naik AD, Kallen MA, Walder A, Street RL Jr. Improving hypertension control in diabetes mellitus: the effects of collaborative and proactive health communication. Circulation 2008;117:1361-8.

18 Elissen A, Nolte E, Knai C, Brunn M, Chevreul K, Conklin A, et al. Is Europe putting theory into practice? A qualitative study of the level of self-management support in chronic care management approaches. BMC Health Serv Res 2013;13:117.

19 Stacey D, Bennett CL, Barry MJ, Col NF, Eden KB, Holmes-Rovner M, et al. Decision aids for people facing health treatment or screening decisions. Cochrane Database Syst Rev 2011;10:CD001431.

20 O'Connor PJ, Desai JR, Butler JC, Kharbanda EO, Sperl-Hillen JM. Current status and future prospects for electronic point-of-care clinical decision support in diabetes care. Curr Diab Rep 2013;13:172-6.

21 Jones JB, Shah NR, Bruce CA, Stewart WF. Meaningful use in practice using patient-specific risk in an electronic health record for shared decision making. Am J Prev Med 2011;40:S179-86.

22 Denig P, Dun M, Schuling J, Haaijer-Ruskamp FM, Voorham J. The effect of a patient-oriented treatment decision aid for risk factor management in patients with diabetes (PORTDA-diab): study protocol for a randomised controlled trial. Trials 2012;13:219.

23 Anderson RM, Funnell MM, Fitzgerald JT, Marrero DG. The Diabetes Empowerment Scale: a measure of psychosocial self-efficacy. Diabetes Care 2000;23:739-43.

24 Horne R, Weinman J, Hankins M. The beliefs about medicines questionnaire: the development and evaluation of a new method for assessing the cognitive representation of medication. Psychol Heal 1999:14:1-24.

25 Pouwer F, Snoek FJ. Patients' Evaluation of the Quality of Diabetes Care (PEQ-D): development and validation of a new instrument. Qual Saf Health Care 2002;11:131-6.

26 Snoek FJ, Pouwer F, Welch GW, Polonsky WH. Diabetes-related emotional distress in Dutch and U.S. diabetic patients. Diabetes Care 2000;231:1305-9.

27 Lamers LM, Stalmeier PF, McDonnell J, Krabbe PF, van Busschbach JJ. [Measuring the quality of life in economic evaluations: the Dutch EQ-5D tariff]. Ned Tijdschr Geneeskd 2005;149:1574-8.

28 Voorham J, Denig P. Computerized extraction of information on the quality of diabetes care from free text in electronic patient records of general practitioners. J Am Med Inform Assoc 2007;14:349-54.

29 Stevens RJ, Kothari V, Adler Al, Stratton IM: United Kingdom Prospective Diabetes Study (UKPDS) Group. UKPDS 56: the UKPDS risk engine: a model for the risk of coronary heart disease in type II diabetes. Clin Sci 2001;101:671-9.

30 Eigenmann CA, Colagiuri R, Skinner TC, Trevena L. Are current psychometric tools suitable for measuring outcomes of diabetes education? Diabet Med 2009;26:425-36.

31 Austin PC. Balance diagnostics for comparing the distribution of baseline covariates between treatment groups in propensity-score matched samples. Stat Med 2009;28:3083-107.

32 Rubin DB. Multiple imputation for non-response in surveys. Wiley, 1987.

33 Branda ME, LeBlanc A, Shah ND, Tiedje K, Ruud K, Van Houten H, et al. Shared decision making for patients with type 2 diabetes: a randomized trial in primary care. BMC Health Serv Res 2013;13:301.

34 Sheehan J, Sherman KA. Computerised decision aids: a systematic review of their effectiveness in facilitating high-quality decision-making in various health-related contexts. Patient Educ Couns 2012;88:69-86. 


\section{What is already known on this topic}

Decision aids can support patient-provider discussions about disease management and encourage shared goal setting and treatment decision making

Most decision aids focus on one specific treatment choice, whereas people with diabetes are often confronted with multiple clinical domains for which treatment is indicated

The effect of the newest generation decision aids that prioritise between domains to guide treatment decisions have not yet been evaluated in a randomised controlled study

\section{What this study adds}

The use of a patient oriented treatment decision aid tackling multiple clinical domains for people with diabetes did not improve patient empowerment by an important amount

The printed version of the decision aid showed limited effects on treatment decisions about lipid regulation

Future studies are needed to evaluate the effects of repeated and flexible use of the decision aid in routine care

35 Linn AJ, Vervloet M, van Dijk L, Smit EG, Van Weert JC. Effects of eHealth interventions on medication adherence: a systematic review of the literature. $J$ Med Internet Res 2011;13:e103.

36 Tang PC, Overhage JM, Chan AS, Brown NL, Aghighi B, Entwistle MP, et al. Online disease management of diabetes: engaging and motivating patients online with enhanced resources-diabetes (EMPOWER-D), a randomized controlled trial. J Am Med Inform Assoc 2013:20:526-34.

37 Ralston JD, Hirsch IB, Hoath J, Mullen M, Cheadle A, Goldberg HI. Web-based collaborative care for type 2 diabetes: a pilot randomized trial. Diabetes Care 2009;32:234-9.

38 Cegala D, Post D, McClure L. The effects of patient communication skills training on the discourse of elderly patients during a primary care interview. J Am Geriatr Soc 2001;49:1505-11.

39 Frost J, Anderson R, Argyle C, Daly M, Harris-Golesworthy F, Harris J, et al. A pilo randomised controlled trial of a preconsultation web-based intervention to improve the care quality and clinical outcomes of diabetes outpatients (DIAT). BMJ Open 2013;3:e003396

40 Ali MK, Shah S, Tandon N. Review of electronic decision-support tools for diabetes care: a viable option for low- and middle-income countries? J Diab Science Technolog 2011;5:553-70.

41 Ramadas A, Quek KF, Chan CK, Oldenburg B. Web-based interventions for the management of type 2 diabetes mellitus: a systematic review of recent evidence. Int $J$ Med Inform 2011:80:389-405.
42 Breslin M, Mullan RJ, Montori VM. The design of a decision aid about diabetes medications for use during the consultation with patients with type 2 diabetes. Patient Educ Couns 2008;73:465-72.

43 Mullan RJ, Montori VM, Shah ND, Christianson TJ, Bryant SC, Guyatt GH, et al. The diabetes mellitus medication choice decision aid: a randomized trial. Arch Intern Med 2009;169:1560-8.

44 Cox K Britten N, Hooper $\mathrm{R}$, White P. Patients' involvement in decisions about medicines: GPs' perceptions of their preferences. Br J Gen Pract 2007;57:777-84.

45 Hajos TR, Polonsky WH, Twisk JW, Dain MP, Snoek FJ. Do physicians understand type 2 diabetes patients' perceptions of seriousness; the emotional impact and needs for care improvement? A cross-national survey. Patient Educ Couns 2011;85:258-63.

\section{Accepted: 28 August 2014}

\section{Cite this as: BMJ 2014;349:95651}

This is an Open Access article distributed in accordance with the Creative Commons Attribution Non Commercial (CC BY-NC 3.0) license, which permits others to distribute, remix, adapt, build upon this work non-commercially, and license their derivative works on different terms, provided the original work is properly cited and the use is non-commercial. See: http://creativecommons.org/licenses/by-nc/3.0/. 


\section{Tables}

\section{Table 1/ Baseline characteristics of participants. Values are numbers (percentages) unless stated otherwise}

\begin{tabular}{|c|c|c|c|}
\hline Characteristics & Intervention $(n=225)$ & Usual care $(n=119)$ & Standardised difference \\
\hline Mean (SD) age (years) & $61.8(8.5)$ & $61.5(8.5)$ & 0.029 \\
\hline Females & $94(42)$ & $54(46)$ & 0.073 \\
\hline Low educational attainment ${ }^{*}$ & $90(40)$ & $45(38)$ & 0.017 \\
\hline \multicolumn{4}{|l|}{ Diabetes duration: } \\
\hline Median (interquartile range) & $6(3)$ & $6(4)$ & \\
\hline$<3$ years & $34(16)$ & $23(20)$ & 0.112 \\
\hline \multicolumn{4}{|l|}{ Drug use: } \\
\hline Insulin & $25(12)$ & $19(16)$ & 0.142 \\
\hline Metformin & $190(85)$ & $102(86)$ & 0.036 \\
\hline Sulphonylurea derivatives & $81(37)$ & $34(29)$ & 0.169 \\
\hline Other glucose regulating drugs & $19(9)$ & $5(5)$ & 0.175 \\
\hline Diuretics & $89(40)$ & $49(42)$ & 0.033 \\
\hline$\beta$ blockers & $72(33)$ & $40(34)$ & 0.025 \\
\hline Calcium antagonists & $40(18)$ & $24(21)$ & 0.061 \\
\hline Angiotensin converting enzyme inhibitors & $95(43)$ & $44(37)$ & 0.107 \\
\hline Angiotensin II receptor blockers & $44(20)$ & $25(21)$ & 0.036 \\
\hline Statins & $174(78)$ & $87(74)$ & 0.098 \\
\hline Other lipid regulating drugs & $10(5)$ & $6(5)$ & 0.028 \\
\hline Related drug use (>3 drugs) & $114(51)$ & $67(57)$ & 0.113 \\
\hline Polypharmacy (>4 drugs for chronic illnesses) & $132(59)$ & $71(60)$ & 0.020 \\
\hline \multicolumn{4}{|l|}{ Risk factors } \\
\hline Uncontrolled HbA1c ( $\geq 7 \%)$ & $72(32)$ & $54(46)$ & 0.277 \\
\hline Mean (SD) HbA1c & $6.8(0.7)$ & $6.8(0.7)$ & 0.088 \\
\hline Uncontrolled SBP ( $\geq 140 \mathrm{~mm} \mathrm{Hg}$ ) & $111(50)$ & $49(42)$ & 0.164 \\
\hline Mean (SD) SBP & $139.1(16.0)$ & $137.1(15.2)$ & 0.129 \\
\hline Uncontrolled LDL ( $\geq 2.5 \mathrm{mmol} / \mathrm{L})$ & $95(43)$ & $45(38)$ & 0.090 \\
\hline Mean (SD) LDL & $2.5(0.9)$ & $2.4(0.8)$ & 0.121 \\
\hline Mean (SD) No of uncontrolled risk factors & $1.4(0.9)$ & $1.4(0.9)$ & 0.041 \\
\hline Microalbuminuria or macroalbuminuria & $25(12)$ & $11(10)$ & 0.157 \\
\hline Smoking & $45(20)$ & $20(17)$ & 0.078 \\
\hline \multicolumn{4}{|l|}{ Patient reported outcomes (mean (SD)) } \\
\hline \multicolumn{4}{|l|}{ Diabetes empowerment scale: } \\
\hline Setting goals & $3.6(0.5)$ & $3.7(0.4)$ & 0.154 \\
\hline Readiness to change & $3.5(0.4)$ & $3.5(0.4)$ & 0.049 \\
\hline Psychosocial management & $3.7(0.5)$ & $3.8(0.4)$ & 0.134 \\
\hline \multicolumn{4}{|l|}{ Beliefs about medication questionnaire: } \\
\hline Necessity & $18.7(3.3)$ & $18.1(3.7)$ & 0.173 \\
\hline Concerns & $14.0(3.6)$ & $14.3(3.5)$ & 0.086 \\
\hline Overuse & $10.6(2.6)$ & $10.5(2.5)$ & 0.032 \\
\hline Harm & $11.5(2.7)$ & $12.0(2.6)$ & 0.108 \\
\hline PEQD & $60.6(19.3)$ & $60.8(19.6)$ & 0.004 \\
\hline Problem area in diabetes & $12.0(13.6)$ & $11.5(12.9)$ & 0.037 \\
\hline EQ5D-NL & $0.86(0.18)$ & $0.91(0.10)$ & 0.304 \\
\hline
\end{tabular}

$\mathrm{SBP}=$ systolic blood pressure; $L D L=$ low density lipoprotein cholesterol; $\mathrm{PEQD}=$ patients' evaluation of quality of diabetes care; EQ5D-NL=Dutch Euro quality of life. *Not more than primary school or lower vocational education. 


\begin{tabular}{|c|c|c|c|c|c|c|}
\hline \multirow[b]{2}{*}{ Outcomes } & \multicolumn{2}{|c|}{ Intervention } & \multicolumn{2}{|c|}{ Usual care } & \multicolumn{2}{|c|}{ Baseline adjusted effect } \\
\hline & No & Mean (SD) & No & Mean (SD) & Effect size $(95 \% \mathrm{Cl})$ & $P$ value \\
\hline \multicolumn{7}{|l|}{ Primary outcome } \\
\hline DES setting and achieving goals & 207 & $3.7(0.5)$ & 108 & $3.7(0.4)$ & $0.039(-0.056$ to 0.134$)$ & 0.424 \\
\hline \multicolumn{7}{|l|}{ Secondary outcomes } \\
\hline \multicolumn{7}{|l|}{ Diabetes empowerment scale: } \\
\hline Readiness to change & 207 & $3.6(0.5)$ & 108 & $3.6(0.5)$ & $-0.017(-0.104$ to 0.071$)$ & 0.710 \\
\hline Psychosocial management & 204 & $3.8(0.5)$ & 108 & $3.8(0.5)$ & $-0.005(-0.094$ to 0.085$)$ & 0.917 \\
\hline \multicolumn{7}{|c|}{ Beliefs about medication questionnaire: } \\
\hline Necessity & 205 & $18.8(3.1)$ & 107 & $18.8(3.3)$ & $-0.314(-0.839$ to 0.210$)$ & 0.241 \\
\hline Concerns & 205 & $14.2(3.4)$ & 107 & $14.0(3.7)$ & $0.442(-0.207$ to 1.092$)$ & 0.183 \\
\hline Overuse & 206 & $10.9(2.5)$ & 108 & $10.5(2.6)$ & $0.385(-0.104$ to 0.874$)$ & 0.124 \\
\hline Harm & 204 & $11.8(2.5)$ & 108 & $11.9(2.6)$ & $0.186(-0.259$ to 0.630$)$ & 0.413 \\
\hline PEQD & 205 & $62.0(19.5)$ & 108 & $62.8(20.6)$ & $-0.728(-4.177$ to 2.721$)$ & 0.679 \\
\hline Problem areas in diabetes & 204 & $12.2(14.5)$ & 107 & $9.9(11.1)$ & $2.115(-0.004$ to 4.234$)$ & 0.051 \\
\hline EQ5D-NL & 203 & $0.88(0.17)$ & 105 & $0.91(0.13)$ & $-0.006(-0.035$ to 0.023$)$ & 0.692 \\
\hline
\end{tabular}

PEQD=Patients' evaluation of quality of diabetes care; EQ5D-NL=Dutch Euro quality of life. 
Table 3 | Drug treatment and smoking status at follow-up, and intervention effects (logistic regression models)

\begin{tabular}{|c|c|c|c|c|c|c|}
\hline \multirow[b]{2}{*}{ Criteria in eligible patients } & \multicolumn{2}{|c|}{ Intervention } & \multicolumn{2}{|c|}{ Usual care } & \multirow[b]{2}{*}{ Odds ratio $(95 \% \mathrm{Cl})$} & \multirow[b]{2}{*}{$P$ value } \\
\hline & No eligible & No (\%) & No eligible & No (\%) & & \\
\hline \multicolumn{7}{|c|}{ Intensified treatments by baseline measures: } \\
\hline Glucose: $\mathrm{HbA} 1 \mathrm{c} \geq 7 \%$ & 68 & $16(24)$ & 51 & $12(24)$ & $1.03(0.44$ to 2.41$)$ & 0.952 \\
\hline Blood pressure: $\mathrm{SBP} \geq 140 \mathrm{~mm} \mathrm{Hg}$ & 107 & $17(16)$ & 48 & $8(17)$ & $0.93(0.37$ to 2.34$)$ & 0.882 \\
\hline Lipids: LDL $\geq 2.5 \mathrm{mmol} / \mathrm{L}$ & 88 & $22(25)$ & 44 & $5(12)$ & 2.54 (0.89 to 7.23$)$ & 0.081 \\
\hline Prescribed RAS inhibiting treatment & 214 & $133(63)$ & 115 & $69(60)$ & $1.27^{\star}(0.46$ to 3.53$)$ & 0.646 \\
\hline Documented smoking status (yes) & 184 & $32(18)$ & 98 & $17(18)$ & $0.40^{\star}(0.04$ to 3.77$)$ & 0.425 \\
\hline
\end{tabular}

$\mathrm{SBP}=$ systolic blood pressure; $\mathrm{LDL}=$ low density lipoprotein cholesterol; RAS=renin angiotensin system .

*Baseline adjusted model. 
Table 4| Process evaluation per intervention format: patient characteristics and reported use of decision aid by patient and healthcare provider. Values are numbers (percentages) unless stated otherwise

\begin{tabular}{|c|c|c|c|c|}
\hline \multirow[b]{2}{*}{ Variables } & \multicolumn{2}{|c|}{ Printed version } & \multicolumn{2}{|c|}{ Screen version } \\
\hline & Short & Extended & Short & Extended \\
\hline Randomised & 53 & 56 & 57 & 59 \\
\hline Mean (SD) age & $60.4(10.0)$ & $61.1(6.7)$ & $64.2(7.0)$ & $61.3(9.6)$ \\
\hline Female & $22(42)$ & $17(31)$ & $27(48)$ & $28(48)$ \\
\hline Diabetes duration $<3$ years & $8(15)$ & $13(24)$ & $3(5)$ & $3(5)$ \\
\hline Mean (SD) UKPDS risk score & $6.3(4.6)$ & $7.4(4.2)$ & $8.0(9.4)$ & $6.1(4.1)$ \\
\hline Mean (SD) number of uncontrolled risk factors & $1.4(0.9)$ & $1.6(0.9)$ & $1.3(0.9)$ & $1.2(0.9)$ \\
\hline Polypharmacy (>4 drugs for chronic illness) & $30(57)$ & $35(63)$ & $38(67)$ & $29(50)$ \\
\hline Intervention consultation planned & $53(100)$ & $53(95)$ & $55(97)$ & $56(95)$ \\
\hline Patient reports & 52 & 49 & 48 & 50 \\
\hline Received information before consultation & $40(77)$ & $41(84)$ & $38(79)$ & $41(82)$ \\
\hline \multicolumn{5}{|l|}{ Information perceived as difficult: } \\
\hline Yes & 2 & 3 & 1 & 4 \\
\hline Neutral & 20 & 7 & 13 & 12 \\
\hline No & 18 & 31 & 24 & 25 \\
\hline \multicolumn{5}{|l|}{ Preference for format } \\
\hline Printed better than screen version & 一 & 一 & 11 & 7 \\
\hline Printed similar to screen version & - & - & 19 & 23 \\
\hline Screen better than printed version & 一 & 一 & 0 & 1 \\
\hline Discussion of risks & $39(75)$ & $41(84)$ & $36(75)$ & $34(68)$ \\
\hline Discussion on possible risk reduction & $40(77)$ & $34(69)$ & $33(69)$ & $27(54)$ \\
\hline Reported intervention as per protocol & $31(60)$ & $25(51)$ & $25(52)$ & $22(44)$ \\
\hline Use of treatment cards & $13(25)$ & $16(33)$ & $20(42)$ & $21(42)$ \\
\hline Provider reports & 43 & 36 & 53 & 53 \\
\hline Patient read information before consultation & $41(95)$ & $36(100)$ & $53(100)$ & $53(100)$ \\
\hline Patient assisted by practice staff & 1 & 0 & 7 & 7 \\
\hline Provider discussed possible risk reduction & $37(86)$ & $30(83)$ & $52(98)$ & $48(91)$ \\
\hline \multicolumn{5}{|l|}{ Provider involved patient in decisions: } \\
\hline Yes & $29(67)$ & $29(81)$ & $37(70)$ & $33(62)$ \\
\hline Not applicable*/missing & $5 / 3$ & $0 / 7$ & $12 / 2$ & $9 / 8$ \\
\hline No & 5 & 0 & 2 & 3 \\
\hline Provider used treatment cards & $0(0)$ & $2(6)$ & $7(13)$ & $8(15)$ \\
\hline Additional consultation planned & 3 & 5 & 6 & 2 \\
\hline
\end{tabular}

UKPDS=United Kingdom Prospective Diabetes Study.

*Risk factors controlled therefore patients' involvement in treatment decisions was unnecessary. 
Table 5| Remarks by healthcare providers about decision aid related to individual patient consultations

\begin{tabular}{lc} 
Classification and description of remark & No of cases \\
Patient positive: & 17 \\
\hline Motivating and/or involved in choice & 10 \\
\hline Confirming and/or reassuring & 5 \\
\hline Useful and/or clear information & 32 \\
\hline Total & \\
\hline Patient concerned or reluctant: & 5 \\
\hline Confronting and/or worrying information & 2 \\
\hline Difficult information & 2 \\
\hline Treatment option not possible & 4 \\
\hline Accepted risks as they were & 13 \\
\hline Total & 7 \\
\hline Patient negative: & \\
\hline Information not accepted or seen as nonsense & 7 \\
\hline Provider reluctant: & 2 \\
\hline No additional value, no action possible & 10 \\
\hline Other priorities for patient, no time for aid & \\
\hline Total & 3 \\
\hline Provider negative: & 3 \\
\hline Not motivating for reducing BMl or stop smoking \\
\hline Practical issues: & 8 \\
\hline Input from medical record not complete / updated & 3 \\
\hline Dutch language, reading glasses needed & \\
\hline Total & \\
\hline
\end{tabular}

BMl=body mass index. 


\section{Figure}

People aged $\leq 65$ years at time Type 2 diabetes diagnosis assessed for eligibility $(n=1769)$

Excluded ( $n=1399)$ :

Not meeting inclusion or meeting exclusion

criteria $(\mathrm{n}=1075)$

Non-response or declined to participate $(n=324)$

Patients consented for study $(\mathrm{n}=370)$

No practice visit during study period $(n=26)$

Randomised $(n=344)$

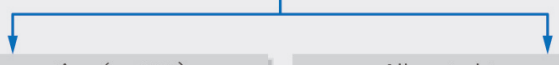

Initial allocation to intervention $(\mathrm{n}=225)$ :

Intervention 1 , screen/short $(n=57)$

Intervention 2, screen/extended $(n=59)$

Intervention 3, printed/short $(n=53)$

Intervention 4, printed/extended $(\mathrm{n}=56)$

Lost to follow-up $(n=4)$ :

Died $(n=1)$

Discontinued study for not wanting or able to

complete questionnaires $(n=3)$

$$
\downarrow
$$

Analysed for primary outcome $(n=199)$

Excluded for missing baseline or follow-up

values in questinnaire $(\mathrm{n}=22)$

Allocated to usual care $(n=119)$

Lost to follow-up $(n=3)$ :

Discontinued study for not wanting or able to complete questionnaires $(n=3)$

Analysed for primary outcome $(n=107)$

Excluded for missing baseline or follow-up

values in questinnaire $(n=9)$

Flow of patients through study 